International Journal of Engineering \& Technology, $7(4.7)(2018) 306-310$
International Journal of Engineering \& Technology
SPC
Website: www.sciencepubco.com/index.php/IJET
Research paper

\title{
Upper Limit Superior and Lower Limit Inferior of Soft Sequences
}

\author{
Ahmed Hasan Hameed ${ }^{1}$, Ekhlas Annon Mousa ${ }^{2}$, Abdulsattar Abdullah hamad ${ }^{3 *}$ \\ ${ }^{1}$ Department of Mathematics Science, Ministry of Education, Babylon \\ Email:amqa92@yahoo.com \\ ${ }^{2}$ Department of Mathematics Science, Ministry of Education, Babylon \\ Email: ekhlasanoon@yahoo.com \\ ${ }^{3}$ Department of Mathematics, College of Science, Tikrit University \\ *Corresponding author E-mail: satar198700@gmail.com
}

\begin{abstract}
In this paper we have provided some of evidence work of the authors R.N.Hasan, O.A Tantawy in 2016 [1] thy given proof of the concept between two bound soft sets \& subsets of soft elements real numbers also was concluded an upper bound and lower bound by using two sequences of soft element real numbers which is

$$
\begin{aligned}
& \left.(I) \sup \left(\tilde{r}_{n} / A \oplus \tilde{i}_{n} / A\right) \tilde{\leq} \sup \tilde{r}_{n} / A \oplus \sup \tilde{i}_{n} / A\right) \\
& (I I) \inf \left(\tilde{r}_{n} / A \oplus \inf \tilde{l}_{n} / A\right) \tilde{\leq} \inf \left(\tilde{r}_{n} / A \oplus \tilde{l}_{n} / A\right)
\end{aligned}
$$

In this paper, supposed to extend R.N.Hasan, O.A Tantawy work but here we are given new notion and proof for upper limit superior and lower limit inferior with two sequences and subsequences for the conclude new proof after recalled that, which is upper limit superior and lower limit inferior By this we have proved above new two theorems and one proposition \& strengthen the example.

AMS Subject Classification: 06D72, 40A05, 54A40
\end{abstract}

Keywords: upper limit superior, lower limit inferior, limit superior, limit inferior, sequences and subsequences, supremum \& infimum

\section{Introduction}

In recent paper R.N.Hasan, O.A.Tantawy 2016 [1] pointed at a concept between two sequences and subsequences clarify the importance and prove the upper bound and lower bound

$$
\begin{aligned}
& (I) \sup \left(\tilde{r}_{n} / A \oplus \tilde{\imath}_{n} / A\right) \tilde{\leq} \sup \left(\tilde{r}_{n} / A \oplus \sup \tilde{l}_{n} / A\right) \\
& (I I) \inf \left(\tilde{r}_{n} / A \oplus \inf \tilde{l}_{n} / A\right) \tilde{\leq} \inf \left(\tilde{r}_{n} / A \oplus \tilde{l}_{n} / A\right)
\end{aligned}
$$

$\mathrm{Bu}$ using supremum \& infimum in [2] 2012 Sujoy Dos and Syamal Kumar Samanta used the concepts soft real numbers \& soft real sets also studied convergence theory of sequences of soft real sets which is limit of soft theory and sequence also given many important notation in same time and in same paper he's studies limit theorem of soft sequences and a generalization soft similarity of two soft real numbers the soft set theoretic approach for dimensionality reduction and supremum \& infimum has potent applications in many different fields which is include the smoothens of functions, game theory, operations research, and in [3] soft set theory is applied to commutatives ideals in BCK-algebra it was suggest the notion of soft limit for function $\mathrm{F}$ at the point see definition (3.3.1) the upper $(\varepsilon, \tau)$-soft limit of function $\mathrm{F}$ at a point $\mathrm{X}$ soft $\bar{t} \lim i t$ and The lower $(\varepsilon, \tau)$-soft limit of function $\mathrm{F}$ at a point $\mathrm{X}$ soft limit, the collection of all these soft limits forms the notions of upper soft limit of the function $\mathrm{F}$ is studied The notions of commutatives soft ideals and commutative idealis- tic soft BCK-algebras are introduced and the notion of positive implicative ideals and introduced their basic properties are derived in [4] M. Irfan Ali Introduce some notion such as the restricted intersection, the restricted difference and the extended intersection of two soft sets also given notion of complement of a soft set, and proved that certain De Morgn's lows hold in soft set theory with respect new definitions.

\section{Preliminaries}

\subsection{Least Upper Bound and Greatest Lower Bound in soft Real Analysis}

Definition 1.1.[1] A soft subset of soft elements Real numbers $F_{A} \subset(R, E)$ is called soft bounded

From above iff there exists a soft Real number

$\tilde{r}=\left\{\left(e,\left\{r_{e}\right\}\right), e \in E\right\}$. Such that

$\tilde{\alpha}=\left\{\left(e,\left\{x_{e}\right\}\right), e \in E\right\} \tilde{\leq} \tilde{r} \forall \tilde{\alpha} \tilde{\in} F_{A}$.

i.e. $\forall e \in E F_{A}(e) \leq r_{e}$

A soft subset of set elements Real numbers $F_{A} \subset(R, E)$

is called soft bounded from below if and only if .

Copyright $\odot 2018$ Authors. This is an open access article distributed under the Creative Commons Attribution License, which permits unrestricted use, distribution, and reproduction in any medium, provided the original work is properly cited. 
There exists a soft Real number $\tilde{r}=\left\{\left(e,\left\{r_{e}\right\}\right), e \in E\right\}$ such that

$$
\beta=\left\{\left(e,\left\{y_{e}\right\}\right), e \in E\right\} \tilde{z} \tilde{r} \forall \tilde{\beta} \tilde{\in} F_{A} .
$$$$
\text { i.e. } \forall e \in E F_{A}(e) \geq \imath_{e}
$$

A soft subset of soft elements Real Numbers

$F_{A} \subset(R, E)$ is called soft bounded if it is both soft bounded From above and below.

Definition 1.2. [1] Let $F_{A} \subset(R, E)$ be a soft subset of soft element real numbers which is bounded from above.

The soft least upper bound $\tilde{r} / A=\left\{\left(e,\left\{r_{e}\right\}\right)\right\}$, of,$F_{A}$ which is denoted by $\sup F_{A}$ is a soft element real number satisfying the following Two condition:

$$
1-\alpha / A \leq r / A \forall \alpha / A \in F_{A}
$$

$$
\text { 2-If } \alpha / A \leq l / A \forall / A \in F_{A} \text {, then } r / A \leq l / A
$$

These two condition can be formulated in another equivalent form to give another equivalent definition For $\sup F_{A}$

Proposition 1.3. [1] For a bounded from above soft subset $F_{A}$ of soft element real number,

If and only if: $\quad r / A=\sup F_{A}$, Iff

$1-\tilde{r} / A$ is an upper bound for $F_{A}$

$2-\forall \in / A=\left\{\left(e_{n},\left\{\in_{e n}\right\}\right)\right\} \check{\check{0}}$ the soft element real number $\tilde{r} / A \oplus \tilde{\varepsilon} / A$ is not upper bound for $F_{A}$.

Proof. Straightforward,Another equivalent form of the definition

Proposition 1.4. [1] For a bounded from above soft subset $F_{A}$ of soft element real number,

$\tilde{r} / A \sup F_{A}$ If and only if.

$1-\tilde{r} / A$ is an upper bound for $F_{A}$.

$2-\forall \tilde{\varepsilon} / A \tilde{\succ} \tilde{0} \exists$ a soft element real number $\tilde{\beta} / A \in F_{A}$ such that $\tilde{\beta} / A \tilde{r} / A{ }^{\Theta} \varepsilon^{Y} / A$ is not an upper bound of $F_{A}$.

Proof. Straightforward

Definition 1.5. [1] For a bounded from above soft subset $F_{A}$ of soft element real number $\sup F_{A} \tilde{\in} F_{A}$

Then we write: $\sup F_{A}=\max F_{A}$

In this case the maximum of the soft real subset $F_{A}$ exist

Remark 1.6. [1] We notice that the soft least upper bound of bounded form above soft subset is one Its upper bounds and therefore we can say that it is the minimum of its upper bounds and write. $\sup F_{A}=\min \left\{\tilde{l} / A: \tilde{\alpha} / A \tilde{\leq} \tilde{l} / A \forall \tilde{\alpha} / A \tilde{\in} F_{A}\right\}$

As a dual of the soft least upper bound of soft subset of soft real number, we have the concept of the greatest soft lower bound.

Definition 1.7. [1] Let $F_{A} \subset(R, E)$ be a soft subset of soft element real numbers with is bounded from below, the soft greatest lower bound $\left.\tilde{m} / A=\left\{e_{a},\left\{m_{e_{a}}\right\}\right)\right\}$, of,$F_{A}$.

which is denoted by: $\tilde{m} / A=\inf F_{A}$.

is a soft element real number satisfying tow conditions: $1-\tilde{\alpha} / A \tilde{\geq} \tilde{m} / A, \forall \tilde{\alpha} / A \tilde{\in} F_{A}$,

2-If $\tilde{\alpha} / A \tilde{z} \tilde{n} / A, \forall \tilde{\alpha} / A \tilde{\in} F_{A}$, Then $\tilde{m} / A \tilde{\geq} \tilde{n} / A$.

These two condition can be formulated in another equivalent form to give another equivalent definition For inf $F_{A}$.

Proposition 1.8. [1] For a bounded from below soft subset $F_{A}$ of soft element real number,

$r / A=\sup F_{A}$, If and only if

$1-\tilde{m} / A, i s$ a lower bound for $F_{A}$,

$2-\forall \in / A=\left\{\left(e_{n},\left\{\in_{e n}\right\}\right)\right\} \check{\succ} \tilde{0}$ the soft element real number

$m / A \oplus \tilde{\varepsilon} / A$ is not a lower bound for $F_{A}$,

Proof. Straightforward Another equivalent form of the definition

Proposition 1.9.[1] For a bounded from below soft subset of soft element real number,

$\tilde{r} / A \sup F_{A}$ If and only if .

$1-\tilde{r} / A$ is a lower bound for $F_{A}$.

$2-\forall \tilde{\varepsilon} / A \tau \tilde{0} \exists$ a soft element real number $\tilde{\beta} / A \in F_{A}$

such that $\tilde{\beta} / A \tilde{>} \tilde{m} / A \oplus \dot{E} / A$ is not an upper bound of $F_{A}$.

Proof. Straightforward

Definition 1.10. [1] For any soft set of soft elements real numbers $F_{A}$ define $\Theta F_{A}$.

$\Theta F_{A}=\left\{-\tilde{\alpha} / A:-\tilde{\alpha} / A \tilde{\in} F_{A}\right\}$.

Proposition 1.12. [1] Let $F_{A} \subset(R, E)$ be a soft subset of soft real numbers which is bounded then $\Theta F_{A}$ is also Bounded and For which we have :

(1) $\sup \ominus F_{A}=-\inf F_{A}$
(2) inf $\ominus F_{A}=-\sup F_{A}$.

Proof: (1) Let $\inf F_{A}=\tilde{m} / A$ them from the equivalent definition of infimum we get: 
(i) $\tilde{m} / A \tilde{\leq} \tilde{\alpha} / A \forall \tilde{\alpha} / A \in F_{A}, A$ nd

(ii) $\forall \tilde{\varepsilon} / A>0, \exists$, asoft, real, number, $\tilde{\beta} / A \in F_{A}$, such, that $\tilde{\beta} / A \tilde{<} \tilde{m} / A \Theta$

$\tilde{\varepsilon} / A$

Multiplying by -1 we get :

(i) $-\tilde{\alpha} / A \tilde{<}-\tilde{m} / A \forall-\tilde{\alpha} / A \in_{\boldsymbol{\theta}} F_{A}$, And

(ii) $\forall \tilde{\varepsilon} / A>\tilde{0}, \exists$, asoft, real, number $-\tilde{\beta} / A \in \oplus F_{A}$, such that $-\tilde{\beta} / A \tilde{>}-m / A$ $\boldsymbol{\Theta}^{\tilde{\varepsilon} / A,}$ (2) is similar to (1).

1.13. [1] Properties of Supremum and Infimum in soft Real Numbers

O.A.Tantawy and R.M. Hassan is given some Pasic properties of supremum and infimum in soft Real Numbers are given in details.

Proposition 1.15. [1] Suppose that $F_{A} \cdot G_{B}$ are non-empty soft sets of soft element real numbers

such that $\tilde{\alpha} \tilde{\leq} \tilde{\beta}$ for all $\tilde{\alpha} \tilde{\in} F_{A}, A n d \tilde{\beta} \tilde{\in} G_{B}$. Then

$F_{A} \tilde{\leq} \inf G_{B}$.

Proof: Fix $\tilde{\beta} \tilde{\in} G_{B}$. since $\tilde{\alpha} \tilde{\leq} \tilde{\beta}$ for all $\tilde{\alpha} \tilde{\in} F_{A}$. it fol-

lows that $\tilde{\beta}$ is an upper bound of $F_{A}$ so, sup $F_{A}$

is finite and $\sup F_{A} \tilde{\leq} \tilde{\beta}$. Hence sup $F_{A}$ is a lower bound of,

$G_{A}$,so inf $G_{A}$ Is finite and $\sup F_{A} \tilde{\leq} \inf G_{A}$.

Definition 1.16. [1] If $F_{A} \tilde{\subset}(R, E)$ and $\tilde{r} \tilde{\in}[R]_{A}^{E}$, then we define

$\tilde{r}_{\Theta} F_{A=\{} \tilde{\imath} \tilde{\in}[R]_{A}^{E}: \tilde{\imath}=\tilde{r}{ }_{\Theta} \tilde{\gamma}$, for.every, $\left.\tilde{\gamma} \tilde{\in} F_{A}\right\}$.

Proposition 1.17. [1] If $\tilde{r} \tilde{\geq} \tilde{0}$, then

$\sup \left(\tilde{r}_{\Theta} F_{A}\right)=\tilde{r} \sup F_{A}, \inf \left(\tilde{r}{ }_{\Theta} F_{A}=\tilde{r} \inf F_{A}\right.$.

If $\tilde{r} \tilde{\leq} \tilde{0}$, then

$\sup \left(\tilde{r}_{\Theta} F_{A}\right)=\tilde{r} \inf F_{A}, \inf \left(\tilde{r}{ }_{\Theta} F_{A}=\tilde{r} \sup F_{A}\right.$.

Proof: the result is obvious if $\tilde{r}=\tilde{0}$. if $\tilde{r}>\tilde{0}$, then $(\tilde{r} \odot$

$\tilde{\gamma}) \tilde{\leq} \tilde{\alpha}$ if and only if $\tilde{\gamma} \tilde{\leq} \tilde{\alpha} / \tilde{r}$,

which show that $\tilde{\alpha}$ is an upper bound of $\tilde{r} \odot F_{A}$, if and only

if $\tilde{\alpha}=\tilde{r}$ is an upper bound of $F_{A}$ so $\sup (\tilde{r}$

$\left.F_{A}\right)=\tilde{r} \sup F_{A} \cdot I f, \tilde{r} \tilde{<} \tilde{0}$, then $\left(\tilde{r}{ }_{\Theta} \tilde{\gamma}_{,} \tilde{\leq} \tilde{\alpha}\right.$.

if then if and only if $\tilde{\gamma} \tilde{z} \tilde{\alpha} / \tilde{r}$, so $\tilde{\alpha}$ is an upper bound of $\tilde{r}$ $\odot F_{A}$,

if and only if $\tilde{\alpha}=\tilde{r}$ is a lower bound of $F_{A}$, so

$$
\sup \left(\tilde{r}_{\Theta} F_{A}\right)=\tilde{r} \inf F_{A} .
$$

the remaining results follow similarly,

Definition 1.18. [1] If $F_{A}, G_{B} \tilde{\subset}(\tilde{R}, E)$, then we define
$F_{A} \oplus G_{B}=\left\{\tilde{r} \tilde{\in}[R]_{\leq E}^{E}: \tilde{r}=\tilde{\alpha} \oplus \tilde{\beta}\right.$ for some

$\left.\tilde{\alpha} \in F_{A}, \tilde{\beta} \in G_{A}\right\}$,

$F_{A}{ }_{\boldsymbol{\theta}} G_{B}=\left\{\tilde{\imath} \tilde{\in}[R]_{\leq E}^{E}: \tilde{\imath}=\tilde{\alpha}{ }_{\boldsymbol{\Theta}} \tilde{\beta}\right.$,for.some

$\left.\tilde{\alpha} \in F_{A}, \tilde{\beta} \in G_{A}\right\}$,

Proposition 1.19. [1] if $F_{A}, G_{B}$, are non-empty soft real sets then,

$\sup \left(F_{A}, G_{B}\right)=\sup F_{A} \oplus \sup G_{B}, \inf \left(F_{A}, G_{B}\right)=\inf F_{A} \oplus \inf G_{B}$.

$\sup \left(F_{A}, G_{B}\right)=\sup F_{A} \quad \Theta \quad \sup G_{B}$,

$\inf \left(F_{A}, G_{B}\right)=\inf F_{A} \sup _{B}$.

Proof; the soft set $F_{A} \oplus G_{B}$ is bounded from above if and only if $F_{A}$ and $G_{B}$ are bounded from above,

So, $\sup \left(F_{A} \oplus G_{B}\right)$, exists if and only if Both $\operatorname{SupF}_{A}$ and $\sup G_{B}$ exist, in that case $\tilde{\alpha} \tilde{\in} F_{A}, A n d, \tilde{\beta} \in G_{A}$, Then, $\tilde{\alpha} \oplus \tilde{\beta} \sup F_{A} \oplus \sup G_{B}$,

So, $\sup F_{A} \oplus \sup G_{B}$, is an upper bound of $F_{A} \oplus G_{B}$, and therefore $\sup \left(F_{A} \oplus G_{B}\right) \tilde{\leq} \sup F_{A} \oplus \sup G_{B}$.

To get the inequality in the opposite directing, suppose that $\tilde{\varepsilon} \sim \tilde{0}$. then there exist $\tilde{\alpha} \tilde{\in} F_{A}$. and $\tilde{\beta} \tilde{\in} G_{B}$ such that

$\tilde{\alpha}>\sup F_{A} \Theta \frac{\tilde{\varepsilon}}{\tilde{2}}, \tilde{\beta}>\sup G_{B} \Theta \frac{\tilde{\varepsilon}}{\tilde{2}}$

It follows that

$\tilde{\alpha} \oplus \tilde{\beta} \tilde{>} \sup F_{A} \oplus \sup G_{B}{ }_{\Theta} \tilde{\varepsilon}$.

For every $\tilde{\varepsilon} \tilde{\mathcal{}} \tilde{0}$, which implies that

$\sup \left(F_{A} \oplus G_{B}\right) \tilde{z} \sup F_{A} \oplus \sup G_{B}$.

Thus,

$\sup \left(F_{A} \oplus G_{B}\right)=\sup F_{A} \oplus \sup G_{B}$.

It follows that

$\sup \left(F_{A} \Theta G_{B}\right)=\sup F_{A} \oplus \sup \left(\Theta G_{B}\right)=\sup F_{A} \ominus$ $\inf G_{B}$.

The proof of the results for $\inf \left(F_{A} \oplus G_{B}\right), A n d, \inf \left(F_{A} \Theta\right.$

$G_{B}$ ) and is similar, or we can apply the results for the supermom to $\Theta F_{A}$ and $G_{B}$

Example 1.20. [1] Let $\left\{\tilde{r}_{n} / A\right\}, A n d\left\{\tilde{\imath}_{n} / A\right\}, n \in N$ be a sequence of soft element real numbers.

Then

(1) $\sup \left(\tilde{r}_{n} / A \oplus \tilde{i}_{n} / A\right) \tilde{\leq} \sup \tilde{r}_{n} / A \oplus \sup \tilde{r}_{n} / A$,

(2)inf $\tilde{r}_{n} / A \oplus \inf \tilde{r}_{n} / A \leq \inf \left(\tilde{r}_{n} / A \oplus \tilde{i}_{n} / A\right)$.

Proof: in fact, let

$\tilde{m} / A=\sup \tilde{r}_{n} / A, n / A=\sup \tilde{l}_{n} / A$, and,$\varepsilon / A=\sup \left(\tilde{r}_{n} / A \oplus \tilde{i}_{n} / A\right)$.

and it is then required to show that:

$\tilde{\varepsilon} / A \leq \tilde{m} / A \oplus \tilde{n} / A$.

From the definition we get 
$\tilde{r}_{n} / A \tilde{\leq} \tilde{m} / A, \forall n$, and $, \tilde{i}_{n} / A \tilde{\leq} n / A, \forall n$

Thus, $\tilde{r}_{n} / A \oplus \tilde{i}_{n} / A \oplus \tilde{n} / A, \forall n \in N$

Hence $\tilde{m} / A \oplus \tilde{n} / A$ is an upper bound for the soft real numbers $\tilde{r}_{n} / A \oplus \tilde{i}_{n} / A$ and consequently is greater than or equal to the least Upper bound $\tilde{\varepsilon} / A$ the proof of (2) is similarly

Proposition 1.21. [1] let $F_{A}$, and,$G_{B}$ bounded soft subset of soft elements real numbers

Such that $F_{A} \subseteq G_{B}$, then we get;

$\inf G_{B} \tilde{\leq} \inf F_{A} \tilde{\leq} \sup F_{A} \tilde{\leq} \sup G_{B}$

Proof: let $G_{B}=\tilde{m}$ then form the definition we get $\tilde{m} \tilde{\leq} \tilde{\alpha}, \forall \tilde{\alpha} \tilde{\in} G_{B}$

Consequently,

$\tilde{m} \tilde{\leq} \alpha, \forall \tilde{\alpha} \tilde{\in} F_{A}$

Hence is a lower bound for the soft subset. It follows then that

Clearly, inf $F_{A} \tilde{\leq} \tilde{\alpha} \tilde{\leq} \sup F_{A} . \forall \tilde{\alpha} \in F_{A}$

Finally, let $\sup G_{B}=\tilde{r}$, then

$\tilde{r} \tilde{\geq} \tilde{\alpha}, \forall \tilde{\alpha} \tilde{\in} G_{B}$ therefore

$\tilde{r} \tilde{\geq} \tilde{\alpha}, \forall \tilde{\alpha} \tilde{\in} F_{A}$

Hence $\tilde{r}$ is an upper bound for the soft subset $F_{A}$, and then $\tilde{r} \geq \sup F_{A}$.

\section{Main Result}

Theorem: let $\left\{X_{n+1}\right\}$ be a soft convergent subsequences of soft real sets converging to soft real sets $X$, then

$\lim \inf \left|<x_{n+1}(\lambda)-<x(\lambda)>\right|=0$ for every $\lambda \in A$.

Proof : since $\mathrm{X}_{\mathrm{n}} \sim \mathrm{X}$ for each $\lambda \in A$ then we can Express that $\limsup \left\langle x_{n+1}(\lambda)\right\rangle=\sup \langle x(\lambda)\rangle$ and also can say

that $\liminf \left\langle x_{n+1}(\lambda)\right\rangle=\inf \langle x(\lambda)\rangle$ for every

$\lambda \in A, \lim \left|\sup <x_{n+1}(\lambda)>-\sup <x(\lambda)>\right|=0$

suppose that $\lambda \in A, \quad \sup <x_{n+1}(\lambda)>=M_{n+1}(\lambda)$ for

all $n \in N$ and $\sup <x(\lambda)>=M(\lambda)$

$\therefore \lim \left|M_{n+1}(\lambda)-M(\lambda)\right|=0$ for arbitrary some $\in>0$ there is a + ve integers $m$ such that $-\in M_{n+1}(\lambda)-M(\lambda)<\in$ for all $n \geq m$, in same way we can choose $x_{n+1}^{\prime}<x_{n+1}(\lambda)>$ and,$x^{\prime} \in<x_{n}(\lambda)>$ Such that

$M_{n+1}(\lambda)-\in / 4<x_{n+1}^{\prime} \leq M_{n}(\lambda)$ and

$M(\lambda)-\in / 4<x^{\prime} \leq M(\lambda)>$

$\therefore-\in-\in / 4\left[M_{n+1}(\lambda)-M(\lambda)\right]$

$-\in / 4 \leq\left[x_{n+1}^{\prime}-x^{\prime} \leq M_{n+1}(\lambda)\right]-M(\lambda)+\in / 4<\in+\in / 4$.

For all $n \geq m$ since $\in>0$ is arbitrary, thus we have $\liminf \left|<x_{n+1}(\lambda)-<x(\lambda)>\right|=0, \forall \lambda \in A$

Remark: if $\left\{x_{n+1}\right\}$ be subsequence of soft real numbers such that $x_{n+1} \rightarrow x$ and $x(\lambda) \neq o, \forall \lambda \in A$ for any $\lambda \in A$ then

$1 l x_{n+1} \sim 1 / x$.

Theorem: suppose $H_{A} . G_{B}$ are non-empty soft sets of soft element real numbers such that

$\tilde{\alpha} \in \beta \quad \forall \tilde{\alpha} \in H_{A}$ and $\tilde{\beta} \in G_{B}$

Let $\tilde{\alpha} \geq \beta, 1-\sup H_{A} \geq \inf G_{B}$ is upper bound,

$2-\inf H_{A} \leq \sup G_{B}$ is lower bound

Proof: clearly $\tilde{\alpha} \tilde{\in} H_{A}$ and also $\tilde{\alpha} \geq \tilde{\beta} \forall \tilde{\beta} \in F_{A}$ it is follows that $\tilde{\alpha}$ is upper bound of $\mathrm{G}_{\mathrm{B}}$ then $\sup \mathrm{G}_{\mathrm{B}}$ is finite and $\sup G_{B} \leq \tilde{\alpha}$,

hence $\sup \mathrm{G}_{B}$ is a lower bound of $\mathrm{H}_{B}$, so inf $\mathrm{H}_{\mathrm{A}}$ is finite and we get that sup $H_{A} \geq \inf G_{A}$.

Proposition: if a $H_{A}, G_{B}$ are non-empty soft real sets then $\lim \sup \left\{H_{A}, G_{B}\right\}(\lambda)=\limsup \left\{H_{A}(\lambda)\right\}+\lim \sup \left\{G_{B}(\lambda)\right\}$

$=\lim \sup \left\{H_{A}(\lambda)+\lim \sup \left\{G_{B}(\lambda)\right\}\right.$

$=\left[\limsup \left\{H_{A}(\lambda)=\sup _{n} \inf _{k \geq n} x_{k}(\lambda)\right]+\left[\lim \left\{G_{B}(\lambda)\right\}=\left[\sup _{n} \inf _{k \geq n} x_{k}(\lambda)\right]\right.\right.$

And

$\liminf \left\{H_{A}, G_{B}\right\}(\lambda)=\liminf \left\{H_{A}(\lambda)\right\}+\liminf \left\{G_{B}(\lambda)\right\}$

$=\liminf \left\{H_{A}(\lambda)\right\}+\liminf \left\{G_{B}(\lambda)\right\}$

$=\left[\liminf \left\{H_{A}(\lambda)=\inf _{n} \sup _{k \geq n} x_{k}(\lambda)\right]+\left[\lim \left\{G_{B}(\lambda)\right\}=\inf _{n} \sup _{k \geq n} x_{k}(\lambda)\right]\right.$

Proof: in a soft sets

$\limsup \left\{H_{A}(\lambda)\right\}+\lim \sup \left\{G_{B}(\lambda)\right\}$ is a bound from

above if and only if $\lim \sup \left\{H_{A}(\lambda)\right\}$ and

$\lim \sup \left\{G_{B}(\lambda)\right\}$ is bound from above it is also should be

$\lim \sup \left\{H_{A}, G_{B}(\lambda)\right\}$ exists if and only if both

$\limsup \left\{H_{A}(\lambda)\right\}$ and $\limsup \left\{G_{B}(\lambda)\right\}$ exists

Here we can say that the case $\tilde{\alpha} \in H_{A}$ And $\tilde{\beta} \tilde{\in} G_{B}$ then $\tilde{\alpha}+\tilde{\beta} \limsup \left\{H_{A}(\lambda)+\lim \sup \left\{G_{B}(\lambda)\right\}\right.$, so $\limsup \left\{H_{A}(\lambda)+\lim \sup \left\{G_{B}(\lambda)\right\}\right.$ is an upper bound of $\left(H_{A}+G_{B}\right)$ and therefore $\lim \sup \left\{H_{A}+G_{B}\right\}(\lambda) \tilde{\leq}$ $\limsup \left\{H_{A}(\lambda)\right\}+\lim \sup \left\{G_{B}(\lambda)\right\}$

Will be getting that inequality in the opposite direct in suppose that $\tilde{\varepsilon} \tilde{\geq} o$, then there exists $\tilde{\alpha} \tilde{\in} H_{A}$ and $\tilde{\beta} \tilde{\in} G_{B}$ such that 
$\tilde{\alpha} \tilde{>} \limsup H_{A}-\frac{\tilde{\varepsilon}}{2}, \tilde{\beta} \tilde{>} \limsup G_{B}-\frac{\tilde{\varepsilon}}{2}$ thus fol-

lows that every $\tilde{\varepsilon} \tilde{\geq} o$, it is implies that $\tilde{\alpha}+\tilde{\beta}$

$\lim \sup \left\{H_{A}(\lambda)+\lim \sup \left\{G_{B}(\lambda)\right\}-\tilde{\varepsilon}\right.$ for every

$\tilde{\varepsilon} \tilde{\geq} o$, that is implies

$\limsup \left\{H_{A}+G_{B}\right\}(\lambda) \geq$

$\limsup \left\{H_{A}(\lambda)\right\}+\lim \sup \left\{G_{B}(\lambda)\right\}$

Thus

$\lim \sup \left\{H_{A}+G_{B}\right\}(\lambda)=\limsup \left\{H_{A}(\lambda)\right\}+\lim \sup \left\{G_{B}(\lambda)\right\}$

We get that $\lim \underline{\sup }\left\{H_{A}-G_{B}\right\}(\lambda)$

$=\limsup \left\{H_{A}(\lambda)\right\}+\lim \sup \left\{G_{B}(\lambda)\right\}$

$=\sup H_{A}-\inf G_{B}$

Example: let $\left\{x_{n} / a\right\},\left\{y_{n} / b\right\} n \in N$ be a two sequence of soft element real numbers then

$1-\limsup _{\underline{m}}\left\{x_{n} / a(\lambda)\right\}+\left\{y_{n} / b(\lambda)\right\} \leq \limsup _{\underline{a}}<\left\{x_{n} / a(\lambda)\right\}+\limsup \left\{y_{n} / b(\lambda)\right\}$

$2-\liminf \left\{x_{n} \mid a(\lambda)\right\}+\liminf \left\{y_{n} \mid b(\lambda)\right\} \leq \liminf \left\langle\left\{x_{n} \mid a(\lambda)\right\}+\left\{y_{n} \mid b(\lambda)\right\}\right\rangle$

Proof: (1) let $\tilde{\imath} / a=\lim \sup \left\{x_{n} / a(\lambda)\right\}$ and

$\tilde{\varphi} / a=\limsup \left\{y_{n} / b(\lambda)\right\}$ and

Let $\tilde{\varepsilon} / a=\limsup _{-}\left\{x_{n} / a\right\}+\left\{y_{n} / b\right\}(\lambda)$ and it is that the required to prove that $\tilde{\varepsilon} / a \tilde{\leq} \tilde{l} / a+\tilde{\varphi} / a$ from the definition we get $\left\{x_{n} / a(\lambda)\right\} \tilde{\leq} \tilde{l} / a, \forall n$, and $\left\{y_{n} / b(\lambda)\right\} \tilde{\leq} \varphi / a$, $\forall n$

Thus is $\left\{x_{n} / a(\lambda)\right\}+\left\{y_{n} / b(\lambda)\right\}+\tilde{\varphi} / a$

$\forall n \in N$.Hence the $\tilde{\imath} / a+\tilde{\varphi} / a$ is an upper bound for the soft real numbers $\left\{x_{n} / a(\lambda)\right\}+\left\{y_{n} / b(\lambda)\right\}$ also and consequently is greater than or equal the least upper bound $\tilde{\varepsilon} / a$ prove (2) is similarly.

\section{References}

[1] Hassan.R.N , Tantawy.OA (2016), Soft Real Analysis, Journal of Progressive Research in Mathematics. vol 8, Issue 1, 1207-1219

[2] Das.S, samanta,SK, (2012) soft real sets, soft real numbers and their properties, Journal of fuzzy mathematics vol.20,NO.3, P 551-576.

[3] Molodtsov.D, (1999), Soft set theory-first results, Compu. Mathematics Applications,Vol.37,issue (4/5), 19-31 Available online https://doi.org/10.1016/S0898-1221(99)00056-5 last visited 16.01. 2018

[4] Jun YB. Kimand HS. Park CH (2011), Positive implicative ideals of BCK-algebras based on soft set theory, Bull.Malays.Math.SciSoc, 34 (2), 345-354

[5] Irfan Ali M, Feng.F, Liu.X, Min WK, and Shabir.M (2009), On some new operations in soft set theory, Computers and Mathematics with Applications, 57(9), 1547-1553 available onlin ehttps://doi.org/10.1016/j.camwa.2008.11.009 last visited 02.02. 2018 